\title{
SUSCEPTIBILIDAD A ANTIBACTERIANOS in vitro DE Salmonella enterica AISLADA DE CUYES DE CRIANZA FAMILIAR-COMERCIAL EN LA PROVINCIA DE CARHUAZ, ÁNCASH
}

\author{
ANTimicrobial in Vitro Susceptibility of Salmonella ENTERICA ISOlated From \\ Guinea Pigs of Familiar-Commercial B reeding Systems in the Province of \\ Carhuaz, Ancash
}

Annie Matsuura S. ${ }^{1}$, Siever Morales C. ${ }^{1,2}$, Sonia Calle E. ${ }^{1}$, Miguel Ara G. ${ }^{3}$

\section{Resumen}

\begin{abstract}
El objetivo del presente estudio fue determinar la sensibilidad de Salmonella enterica aislada de cuyes, con signos clínicos sugerentes de salmonelosis, frente a 12 antibacterianos. Los cuyes fueron criados bajo un sistema familiar-comercial en la provincia de Carhuaz, Áncash, entre mayo a julio de 2007. Se colectó muestras de bazo, hígado y otros órganos con lesión aparente $(\mathrm{n}=65)$. Para el aislamiento de $S$. enterica se utilizaron métodos convencionales y el análisis de sensibilidad a antibacterianos fue mediante el método de difusión por discos de Kirby-Bauer. Cepas de S. enterica se aislaron en el $61.5 \%$ de los cuyes. El $100 \%$ de estas cepas (40/40) fue sensible a enrofloxacina, sulfatrimetoprim, estreptomicina y amoxicilina, el $97.5 \%$ al cloranfenicol y gentamicina, y el $92.5 \%$ a fosfomicina. Se encontraron cepas resistentes a furazolidona $(15.0 \%)$ y colistina $(12.5 \%)$ entre otras. Los resultados indican que la sensibilidad de $S$. enterica a enrofloxacina y sulfatrimetoprim, así como al cloranfenicol, gentamicina y fosfomicina, hacen de estos antibacterianos buenas alternativas para el tratamiento de la salmonelosis en las granjas estudiadas.
\end{abstract}

Palabras clave: Salmonella enterica, cuyes, antibacterianos, susceptibilidad

\section{Abstract}

The objective of the present study was to determine the sensitivity of Salmonella enterica to 12 antibiotics. The bacteria were isolated from guinea pigs showing clinical signs compatible to salmonellosis. Samples of spleen, liver and other organs showing pathological lesions were collected from unhealthy guinea pigs bred under a familiarcommercial system in the province of Carhuaz, Ancash between May to July 2007. Conventional methods were used in order to isolate $S$. enterica strains and the diffusion test of Kirby-Bauer method was used to evaluate the sensitivity to antibiotics. Strains of

\footnotetext{
${ }^{1}$ Laboratorio de Microbiología y Parasitología Veterinaria, ${ }^{3}$ Laboratorio de Bioquímica, Nutrición y Alimentación Animal, Facultad de Medicina Veterinaria, Universidad Nacional Mayor de San Marcos, Lima

${ }^{2}$ E-mail: sieverm@gmail.com
} 
S. enterica were isolated from $61.5 \%$ of the animals. All of these strains (40/40) were sensible to enrofloxacin, sulfatrimethoprim, streptomycin, and amoxicillin, $97.5 \%$ to chloramphenicol and gentamicin, and $92.5 \%$ to fosfomicin. Resistant strains were found to furazolidone $(15.0 \%)$ and colistin $(12.5 \%)$ among others. The results showed that sensitivity to enrofloxacin and sulfatrimethoprim, as well as chloramphenicol, gentamicin and fosfomicin indicated these antibiotics are good choices for salmonelosis treatment in the studied farms.

Key words: Salmonella enterica, guinea pig, antibiotics, susceptibility

\section{INTRODUCCIÓN}

El cuy o cobayo constituye una fuente proteica esencial en la dieta del poblador andino, y en las últimas décadas se ha convertido en un producto de mayor demanda por el mercado nacional e internacional (Chauca, 1994). El Perú cuenta con una población aproximada de 23 millones de cuyes, con una distribución de $92 \%$ en la sierra, $6 \%$ en la costa y $2 \%$ en la selva (MINAG, 2003); siendo Áncash el tercer departamento con mayor población de cuyes (INEI, 1996).

La crianza del cuy se encuentra aún en mayor medida en áreas rurales bajo un sistema familiar-comercial, fuertemente limitada por aspectos de manejo y sanidad, siendo la salmonelosis la enfermedad más importante (Bustamante, 1993). Es causada por Salmonella enterica, bacilo gramnegativo intracelular facultativo, de la familia Enterobacteriaceae (Figueroa y Verdugo, 2005). Esta bacteria se encuentra como patógeno en el tracto gastrointestinal de mamíferos, reptiles, aves e insectos, causando un amplio espectro de enfermedades en el hombre y los animales (Terragno et al., 2003).

La infección se relaciona con estrés, malas prácticas de manejo y el deficiente nivel de bioseguridad, como es la presencia de roedores y aves, y el ingreso no controlado del personal que permiten la contaminación de ambientes y alimentos (Figueroa y Verdugo, 2005). Las variaciones de temperatura y humedad predisponen a la infección (Ramírez, 1972).
Los signos clínicos en el cuy se manifiestan en forma aguda o crónica. La forma aguda produce altos índices de mortalidad en la población, en un curso de 24 a 48 horas. En los casos crónicos es notoria la caquexia, anorexia, pelaje hirsuto, diarrea, debilidad, parálisis de los miembros, neumonía, abortos, $\mathrm{y}$ abdomen hinchado (Morales et al., 2007). El diagnóstico de la enfermedad es a través del aislamiento del microorganismo mediante cultivo bacteriológico en el laboratorio (Haavelar et al., 2001).

La efectividad de los tratamientos se ve afectada por la falta de un diagnóstico correcto y la generación de resistencia de la bacteria a los antibióticos, por lo que la elección del agente terapéutico debe estar basada en pruebas de susceptibilidad (Mateu y Martin, 2001). El uso excesivo e inapropiado de antibacterianos es el factor más importante en la aparición y diseminación de la resistencia (Mejía, 2003). En base a estos antecedentes, se diseñó el presente estudio para determinar el patrón de susceptibilidad antibacteriana de las cepas de Salmonella entérica aislada de cobayos de la provincia de Carhuaz.

\section{Materiales y Métodos}

\section{Lugar de Estudio}

El estudio se realizó en varios centros de crianza de tipo familiar-comercial de la provincia de Carhuaz, Áncash, en el periodo de mayo a julio del 2007, y el análisis bacteriológico se hizo en el Laboratorio de Micro- 
biología de la Facultad de Medicina Veterinaria de la Universidad Nacional Mayor de San Marcos, Lima.

Se seleccionó cuyes que presentaron signos clínicos de salmonelosis. Los animales se estratificaron en base a su edad como gazapos (en lactación), recría (del destete al empadre) y adultos. Se registró la historia clínica y evaluación externa, y luego se procedió a la evaluación interna, observación de los órganos y toma de muestras de hisopados de hígado, bazo y órganos con lesiones. Las muestras se conservaron en medio de transporte Stuart (Merck) a $4{ }^{\circ} \mathrm{C}$.

La siembra de los hisopados se hizo en medios de cultivo agar McConkey, incubándose por $48 \mathrm{~h}$ a $37^{\circ} \mathrm{C}$. Se subcultivó el hisopado en caldo de enriquecimiento tetrationato (CT), y se repicó en medio selectivo agar Xilosa-Lisina-Desoxicolato (XLD) por $24-48 \mathrm{~h}$ a $37^{\circ} \mathrm{C}$. Las colonias sospechosas de Salmonella sp. se cultivaron en agar McConkey. En este medio, las colonias son incoloras, mientras que en agar XLD son rojas con un centro negro (Pasterán et al., 2003). Las colonias fueron identificadas mediante pruebas bioquímicas y de serología en aglutinación en placa.

La susceptibilidad a antibacterianos se analizó mediante el método de difusión de discos o técnica de Kirby-Bauer. Las cepas aisladas se suspendieron en solución salina fisiológica ajustándolas a una turbidez de $0.5 \mathrm{del}$ estándar de McFarland y luego fueron sembradas en agar Mueller-Hinton donde se enfrentaron a 12 antibacterianos: colistina (10 $\mu \mathrm{g})$, cloranfenicol $(30 \mu \mathrm{g})$, doxiciclina $(30 \mu \mathrm{g})$, enrofloxacina $(5 \mu \mathrm{g})$, neomicina $(30 \mu \mathrm{g})$, amoxicilina $(25 \mu \mathrm{g})$, oxitetraciclina $(30 \mu \mathrm{g})$, gentamicina $(10 \mu \mathrm{g})$, sulfatrimetoprim $(25 \mu \mathrm{g})$, estreptomicina $(10 \mu \mathrm{g})$, fosfomicina $(200 \mu \mathrm{g})$ y furazolidona $(100 \mu \mathrm{g})$. Los antibacterianos se eligieron en base a su eficacia, disponibilidad en el mercado, y toxicidad. Las placas se incubaron a $37^{\circ} \mathrm{C}$ por $24 \mathrm{~h}$. El halo de inhibición se interpretó como cepas sensibles, intermedias o resistentes (NCCLS, 1999).

\section{Resultados y Discusión}

Se evaluaron muestras de tejidos de 65 cuyes con signos clínicos de enfermedad tales como debilidad (30/65), caquexia (24/65), anorexia (21/65), aislamiento $(18 / 65)$, pelo hirsuto (15/65), abdomen hinchado (14/65), muerte repentina $(14 / 65)$, diarrea $(9 / 65)$, aborto $(5 / 65)$, infertilidad $(2 / 65)$ y parálisis del tren posterior $(1 / 65)$.

El 61.5\% (40/65) estaba infectado con Salmonella enterica. Este alto índice de presentación de la infección es influenciado por factores como la virulencia del microorganismo, la condición del hospedero y el medio ambiente. La intensidad de la infección va a depender del serotipo, la dosis infectiva, y el estado inmunológico del animal; es así que animales expuestos a dosis de $10^{6}-10^{8}$ bacterias desarrollan la enfermedad sintomática (Jubb y Palmer, 1991). Asimismo, la bacteria debe vencer barreras naturales en el hospedero como el $\mathrm{pH}$ ácido, a través de factores de virulencia que son regulados por genes localizados en islas de patogenicidad (SPIs) y a sistemas regulatorios como el PhoPQ, que confieren la capacidad a la bacteria para sobrevivir y multiplicarse intracelularmente en los macrófagos (Goyache y Briones, 2002; Amsterdam et al., 2004).

Entre los 40 cuyes infectados, la etapa de recría fue la más afectada $(52.5 \%, 21 /$ $40)$, seguido de los adultos $(32.5 \%, 13 / 40)$, mientras los lactantes representaron el 15\% (6/40) de los animales positivos. Otros estudios demuestran que cuyes en la etapa de crecimiento o post destete son los más afectados (S. Morales, Lima, datos no publicados). Por otro lado, las hembras fueron el grupo más afectado $(75 \%, 30 / 40)$.

Los órganos donde se aisló la bacteria con mayor frecuencia fueron el bazo $(92.5 \%$, $37 / 40)$ y el hígado (87.5\%, 35/40). El bazo ha sido reportado como el órgano más sensible y útil para la detección de Salmonella enterica en infecciones sistémicas aislada 
Cuadro 1. Distribución de la susceptibilidad (\%) a antibacterianos de Salmonella enterica aislada de cuyes clínicamente enfermos durante el periodo mayo-julio 2007 en la provincia de Carhuaz, Áncash ( $\mathrm{n}=40$ )

\begin{tabular}{lccc}
\hline Antibacteriano & Sensible & Intermedio & Resistente \\
\hline Enrofloxacina & 100.0 & 0 & 0 \\
Sulfatrimetoprim & 100.0 & 0 & 0 \\
Estreptomicina & 100.0 & 0 & 0 \\
Amoxicilina & 100.0 & 0 & 0 \\
Cloranfenicol & 97.5 & 2.5 & 0 \\
Gentamicina & 97.5 & 2.5 & 0 \\
Fosfomicina & 92.5 & 7.5 & 0 \\
Furazolidona & 82.5 & 2.5 & 15.0 \\
Colistina & 72.5 & 15.0 & 12.5 \\
Doxiciclina & 72.5 & 20.0 & 7.5 \\
Neomicina & 70.0 & 25.0 & 5.0 \\
Oxitetraciclina & 50.0 & 42.5 & 7.5 \\
\hline
\end{tabular}

Cuadro 2. Distribución de aislamientos de Salmonella enterica en órganos de cuyes clínicamente enfermos

\begin{tabular}{lccc}
\hline \multirow{2}{*}{ Órgano } & $\begin{array}{c}\text { Número de } \\
\text { muestras }\end{array}$ & \multicolumn{2}{c}{ Casos positivos de Salmonella enterica } \\
\cline { 3 - 4 } & 40 & $\mathrm{~N} .^{\circ}$ & $\%$ \\
\hline Hígado & 40 & 35 & 87.5 \\
Bazo & 24 & 37 & 92.5 \\
Pulmón & 15 & 19 & 79.2 \\
Útero & 5 & 14 & 93.3 \\
Vesícula biliar & 15 & 2 & 40.0 \\
Intestino & 2 & 12 & 80.0 \\
Ganglio mesentérico & 1 & 1 & 50.0 \\
Glándula mamaria & & 1 & 100.0 \\
\hline
\end{tabular}

en roedores (Havelaar et al., 2001). Esto se explica porque la bacteria invade las células $\mathrm{M}$ del intestino, sobreviviendo intracelularmente, para de allí comenzar su diseminación vía linfática y sanguínea hacia el hígado y bazo, donde sigue multiplicándose (Figueroa y Verdugo, 2005). Asimismo, en el presente estudio se aisló S. enterica del pulmón (19/24), 
útero $(14 / 15)$, intestino $(12 / 15)$, vesícula biliar (2/5), ganglio mesentérico (1/2) y glándula mamaria $(1 / 1)$.

El $100 \%$ de las 40 cepas de S. enterica aisladas eran sensibles a cuatro drogas: enrofloxacina, sulfatrimetoprim, estreptomicina y amoxicilina. Asimismo, un alto porcentaje fue sensible al cloranfenicol y gentamicina $(97.5 \%)$ y a fosfomicina (92.5\%) (Cuadro 1$)$.

Además, se encontraron cepas resistentes a furazolidona $(15.0 \%)$ y colistina $(12.5 \%)$ entre otras. Por otro lado, el $42.5 \%$ de las cepas mostraron una susceptibilidad intermedia contra oxitetraciclina (Cuadro 1).

Los resultados de susceptibilidad de la S. entérica contra enrofloxacina y sulfatrimetoprim fueron buenos; sin embargo, se presume un aumento de la resistencia a este antibacteriano considerando el hecho de su uso frecuente en la zona contra problemas infecciosos en cuyes, aves y otras especies domésticas (Morales, Lima, comunicación personal).

El mejor antibiótico in vitro puede no ser siempre la mejor opción, dado los efectos tóxicos inducidos por algunos antibióticos. Es así, que a pesar de la sensibilidad alcanzada para amoxicilina, el uso de este antibiótico en la producción de cuyes no es recomendable debido a que las penicilinas, los macrólidos y las lincosamidas son tóxicas en cuyes. Estos antibacterianos pueden causar cambios en el $\mathrm{pH}$ al suprimir la flora grampositiva del intestino del cuy, permitiendo el desarrollo de bacterias gramnegativas, que son potencialmente patógenas en esta especie, produciendo enterocolitis y muerte (Serevova, 1964; Quesenberry, 1994).

Las diferencias en cepas resistentes entre los aminoglucósidos estreptomicina y gentamicina con neomicina, se puede explicar porque las enzimas bacterianas empleadas que las inactivan pueden ser específicas para cada uno de ellos (Harkness, 1995; Morris, 1995).
El uso de cloranfenicol y furazolidona ha sido prohibido en la Unión Europea y EEUU en animales de consumo por las graves implicaciones clínicas en humanos y sus propiedades carcinogénicas en roedores, respectivamente (Chadfield y Hinton, 2004; Intorre et al., 2005). Sin embargo, en el Perú se siguen empleando, hecho que debe prestarse especial atención.

La sensibilidad intermedia y resistencia a la tetraciclina encontrada no es extraña debido al uso común de este antibacteriano. Esta resistencia puede ser transmitida a través de plásmidos que codifican mecanismos bacterianos como bombas de flujo (Escolar et al., 1998). La resistencia suele ser cruzada entre los distintos componentes de esta familia de fármacos, hecho que puede ser corroborado en este estudio al observar los porcentajes de susceptibilidad intermedia y de resistencia frente a doxiciclina (Cuadro 1).

La colistina se había constituido en una alternativa para el tratamiento de este tipo de infecciones. Se emplea en la industria porcina española y se reporta una baja frecuencia de resistencia (Mejía, 2003); sin embargo, ya no es utilizada en humanos para el tratamiento de la salmonelosis debido a la generación de resistencia (INFOSAN, 2005).

Se debe tener presente que el aislamiento de la bacteria procedente de diferentes especies animales o región geográfica puede mostrar un distinto patrón de resistencia a antibacterianos (Wasyl y Hoszowski, 2004), pudiendo depender de los hábitos locales en el uso de antibacterianos, por lo que los resultados observados no son extrapolables a Salmonella que se aíslen en otras áreas del país. Debido a esto, se recomienda que cada centro de producción evalúe periódicamente los patrones de resistencia de las bacterias aisladas en su ambiente a fin de determinar las pautas de la terapia antibacteriana a seguir. 


\section{Conclusiones}

? La susceptibilidad de Salmonella enterica a enrofloxacina y sulfatrimetoprim, así como al cloranfenicol, gentamicina y fosfomicina, hacen de estos antibacterianos buenas alternativas de elección para el tratamiento de la salmonelosis en las granjas bajo estudio.

? S. enterica fue el microorganismo más frecuentemente aislado en cuyes clínicamente enfermos en la provincia de Carhuaz durante el periodo mayo-julio de 2007.

\section{Agradecimientos}

Los autores agradecen a la ONG ADG (Aide au Développement Gembloux, Bélgica), y BIDA (Centro de Biodiversidad y Desarrollo Agrario La Molina - Perú) por el financiamiento de estudio; y a Marie Smekens (ONG ADG) por la coordinación y establecimiento del proyecto.

\section{Literatura Citada}

1. Amsterdam L, De Jong W, De Jonge R, Hoebee B. 2004. Genetic susceptibility for Salmonella infections. RIVM report. [Internet], [08 agosto 2007]. Disponible en: http://www.rivm.nl/ bibliotheek/rapporten/340210001.html

2. Bustamante J. 1993. Producción de cuyes. Lima: Facultad de Medicina Veterinaria de la Universidad Nacional Mayor de San Marcos. 259 p.

3. Chadfield M, Hinton M. 2004. In vitro activity of nitrofuran derivatives on growth and morphology of Salmonella enterica serotype Enteritidis. J Appl Microbiol 96: 1002-1012.

4. Chauca L. 1994. Sistemas de Producción de cuyes. En: Crianza de cuyes. Lima: Instituto Nacional de Investigación Agraria - INIA. Serie didáctica. 170 p.
5. Escolar M, Azanza J, Sádaba B, Honorato J. 1998. Tetraciclinas, cloranfenicol y fosfomicina. Medicine 7: 3524-3532.

6. Figueroa I, Verdugo A. 2005. Mecanismos moleculares de patogenicidad de Salmonella sp. Rev Latinoam Microbiol 47: 25-42.

7. Goyache J, Briones V. 2002. Géneros Salmonella y Shiguella. En: Vadillo S, Piriz M. Manual de microbiología veterinaria. Cap 22. Madrid: McGraw-Hill. p 327-338.

8. Harkness J. 1995. Rodent drug dosages. En: Bauck L, Boyer L, Brown S, Harkness J, Jenkins J (eds). Exotic animal formulary: a supplement to AAHA's practitioner guides to exotic animal medicine. Colorado: American Animal Hospital Association. p 37-46.

9. Havelaar A, Garssen T, Takumi K, Koedam M, Dufrenne J. 2001. A rat model for dose-response relationships of Salmonella enteritidis infection. J Appl Microbiol 91: 442-452.

10. [INEI] Instituto Nacional de Estadística e Informática. 1996. III Censo Nacional Agropecuario. [Internet], [22 setiembre 2007]. Disponible en: www.iinei.inei.gob.peliinei/ cenagro1994/

11. [INFOSAN] Red Internacional de Autoridades de Inocuidad de los alimentos. 2005. Resistencia antimicrobiana a Salmonella. Ginebra: OMS. [Internet], [19 diciembre 2007]. Disponible en: ww w . who.int/foodsafety/ fs_management/ No_03_Salmonella_ Apr05_sp.pdf

12. Intorre L, Vanni M, Ebani V, Cerri D, Fratini F. 2005. Antimicrobial susceptibility of animal strains of Salmonella enterica isolated in Italy from 2001 to 2003. J Vet Pharmacol Ther 28: $121-125$.

13. Jubb P, Palmer N. 1991. Patología de los animales domésticos. $3^{\mathrm{a}}$ ed. Uruguay: Agropecuaria Hemisferio Sur. p 160-170.

14. Mateu E, Martin M. 2001. Why is antimicrobial resistance a veterinary problem as well? J Vet Med B 48: 569-581. 
15. Mejía W. 2003. Epidemiología de la salmonelosis porcina en granjas de Cataluña y determinación de los factores de riesgo de la infección. Tesis doctoral. Barcelona: Facultad de Veterinaria de la Universidad Autónoma de Barcelona. 99p.

16. [MINAG] Ministerio de Agricultura del Perú. 2003. Realidad y problemática del sector pecuario- Cuyes. [Internet], [09 enero 2008] Disponible en: http:// www.minag.gob.pe/pecuaria/ pec_crianza_produccion_cuyes.shtml

17. Morales S, Mattos J, Calle S. 2007. Efecto de la muña (Satureja parvifolia) en la dinámica de la infección por Salmonella enterica en cobayos. En: XXX Reunión Científica Anual Asociación Peruana de Producción Animal. Cuzco-Perú: APPA.

18. [NCCLS] National Committee for Clinical Laboratory Standards. 1999. Performance standards for antimicrobial disk and dilution susceptibility test for bacteria isolated from animals. Approved Standard. $2^{\text {nd }}$ ed. USA: NCCLS Document M31-A2. $82 \mathrm{p}$.

19. Morris T. 1995. Antibiotic therapeutics in laboratory animals. Lab Anim 29: 16-36.
20. Quesenberry K. 1994. Guinea pig. Vet Clin N Am 24: 25-64.

21. Pasterán F, Corso A, Galas M. 2003. Salmonella: sensibilidad a los antimicrobianos. En: Manual de procedimientos. Argentina: Instituto Nacional de Enfermedades Infecciosas - ANLIS "Dr. Carlos G. Malbrán". 32 p.

22. Ramirez I. 1972. Estudio bacteriológico y epidemiológico de un brote infeccioso en cobayos (Cavia porcellus). Tesis Médico Veterinario. Lima: Univ. Nacional Mayor de San Marcos. 62 p.

23. Severova Y. 1964. Dynamics of morphological changes in guinea pig organs after injection of penicillin. Antibiotiki 9: 44-49.

24. Terragno $R$, Caffer $M$, Bruno $S$, Binsztein N. 2003. Salmonella: aislamiento, identificación y serotipificación. En: Manual de Procedimientos. Argentina: Instituto Nacional de Enfermedades Infecciosas - ANLIS “Dr. Carlos G. Malbrán”. 46 p.

25. Wasyl D, Hoszowski A. 2004. Antimicrobial resistance of Salmonella isolated from animals and feed in Poland. Bull Vet Inst Pulawy 48: 233-240. 